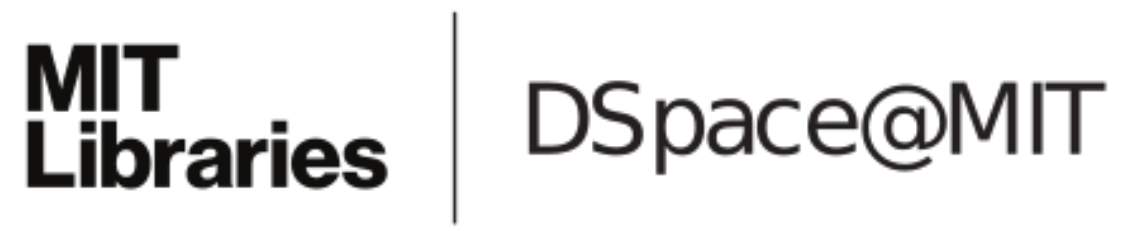

\author{
MIT Open Access Articles
}

Time-scale separation based design

of biomolecular feedback controllers

The MIT Faculty has made this article openly available. Please share how this access benefits you. Your story matters.

Citation: Grunberg, Theodore W. and Del Vecchio, Domitilla. 2019. "Time-scale separation based design of biomolecular feedback controllers." Proceedings of the IEEE Conference on Decision and Control, 2019-December.

As Published: $10.1109 /$ cdc40024.2019.9029355

\section{Publisher: IEEE}

Persistent URL: https://hdl.handle.net/1721.1/137975

Version: Author's final manuscript: final author's manuscript post peer review, without publisher's formatting or copy editing

Terms of use: Creative Commons Attribution-Noncommercial-Share Alike 


\title{
Time-scale separation based design of biomolecular feedback controllers
}

\author{
Theodore W. Grunberg ${ }^{1}$ and Domitilla Del Vecchio ${ }^{2}$
}

\begin{abstract}
Time-scale separation is a powerful property that can be used to simplify control systems design. In this work, we consider the problem of designing biomolecular feedback controllers that provide tracking of slowly varying references and rejection of slowly varying disturbances for nonlinear systems. We propose a design methodology that uses timescale separation to accommodate physical constraints on the implementation of integral control in cellular systems. The main result of this paper gives sufficient conditions under which controllers designed using our time-scale separation methodology have desired asymptotic performance when the reference and disturbance are constant or slowly varying. Our analysis is based on construction of Lyapunov functions for a class of singularly perturbed systems that are dependent on an additional parameter that perturbs the system regularly. When the exogenous inputs are slowly varying, this approach allows us to bound the system trajectories by a function of the regularly perturbing parameter. This bound decays to zero as the parameter's value increases, while an inner-estimate of the region of attraction stays unchanged as this parameter is varied. These results cannot be derived using standard singular perturbation results. We apply our results to an application demonstrating a physically realizable parameter tuning that controls performance.
\end{abstract}

\section{INTRODUCTION}

Modular composition of genetic circuits, wherein a circuit's functionality is unaltered by the presence of surrounding circuits, is critical to design increasingly sophisticated systems [1]-[3]. However, modular composition is challenged by a number of factors, including loading effects due to direct connectivity and indirect connectivity due to resource sharing [4], the fact that different modules use the same pool of cellular resources, which causes unintended interactions between modules [5], [6]. One approach to making biomolecular modules robust to the context in which they are placed is to utilize feedback control within a module such that the output of the module rejects disturbances such as fluctuations in available resources [7]. While this is a promising approach, it requires designing biomolecular controllers capable of reference tracking and disturbance rejection, i.e. making the behavior of a module depend only on its intended input, not on any disturbances arising from changing context.

Several control architectures have been proposed in the design of genetic circuits, such as those based on transcriptional autorepression [8], covalent modification [9], and

This work was supported in part by NSF-CMMI grant 1727189 .

${ }^{1} \mathrm{~T}$. W. Grunberg is with the Department of Electrical Engineering and Computer Science, MIT, grunberg@mit . edu

${ }^{2} \mathrm{D}$. Del Vecchio is with the Department of Mechanical Engineering, MIT, ddv@mit.edu sequestration [7], [10]-[12]. Thus far the design of biomolecular feedback controllers has focused on two properties: making the location of an equilibrium point of the closed loop system insensitive to disturbances and track constant inputs, and ensuring that this equilibrium point is locally asymptotically stable, with many studies including both criteria [10], [13]-[15]. For a large class of systems, integral control is necessary for zero steady state error when constant disturbances are applied [16]. In the theoretical scenario where molecules do not dilute due to cell growth, exact implementations of integral action are possible [10], [17]. However, in growing and dividing cells, the volume in which the reactions occur increases with time, and therefore all species except those such as DNA, which is specifically regulated by the cell's replication mechanism, are affected by dilution. Despite this, there are circuit architectures that, due to high gain feeback based on time-scale separation between fast and slow chemical reactions, can recover almost perfect adaptation even in the presence of molecular dilution [13] However, in this case it is not generally possible to conclude that the equilibrium point is locally asymptotically stable uniformly in the gain parameters which control the location of the equilibrium point. Therefore, in this work we introduce a framework based on Lyapunov analysis which can guarantee asymptotic tracking and disturbance rejection for slowly varying reference and disturbance signals, in the limit as a regular perturbation parameter goes to infinity. We demonstrate how for important biomolecular controller motifs, our results can be used to show that asymptotic tracking and disturbance rejection can be guaranteed by simple-to-check conditions on the system. We apply these results to a feedback control system implemented in bacterial cells with mRNA-sRNA interference [7].

Related work: Reference tracking problems similar to the one we consider in this work have been studied previously in many contexts. In a large class of reference tracking problems involving high gain feedback, there is a transformation of the system into standard singular perturbation form [18] [20]. However, in biologically relevant systems including those which are known to asymptotically perfectly adapt to disturbances, the system cannot be written in standard singular perturbation form with respect to the parameters controlling the feedback gain [21]. Therefore, the previously derived results are not applicable. Additionally, the synthesis of controllers for reference tracking in nonlinear systems has been studied extensively, e.g. [22]-[24]. However, in biomolecular systems it is impossible to precisely implement arbitrary controllers, and therefore techniques which certify tracking performance only when the controller synthesized 
by the given procedure is used cannot be applied. Instead, we seek analysis techniques which can aid the design process by certifying performance for given human designed controllers.

The rest of this paper proceeds as follows. In Section II we introduce the system model that we study and give a formal definition of the behavior that we desire from the closed loop system, which we call asymptotic robust reference tracking. In Section III we give results that show how Lyapunov functions for the boundary and reduced systems can be composed to ensure asymptotic robust reference tracking of the closed loop system. In Section IV we then give a biologically relevant class of systems where asymptotic robust reference tracking can be certified. In Section V we apply these results to a protein production process with a mRNA-sRNA interference based controller. Conclusions and directions for future work are discussed in Section VI.

\section{PROBLEM SETTING}

Our approach is to first design controllers that have desirable equilibrium input-output characteristics, and then create time-scale separation between the plant and controller to guarantee reference tracking and disturbance rejection of slowly varying exogenous inputs. This two-step approach allows us to obtain formal guarantees on the robust performance of the closed loop system. Additionally, it allows us to make use of previously known robustness properties of the equilibrium point of certain closed loop systems that have been previously analyzed, see e.g. [13]. We consider a system composed of two blocks, the plant, $P$, which is assumed to be specified a priori, and the controller, $C$, which is to be designed to give the closed loop system the desired properties. A key assumption of our work is that the controller has two design parameters, $\epsilon$ and $\lambda$. Parameter $\epsilon$ singularly perturbs the closed loop dynamics by making the controller dynamics become faster as $\epsilon \rightarrow 0$, and thus under stability conditions the closed loop dynamics approach those of the reduced system obtained by setting $\epsilon=0$. Parameter $\lambda$, in turn, is such that as $\lambda$ goes to infinity, the steady state output of the system becomes closer to the reference, $r$, and becomes insensitive to a disturbance, $d$. Additionally, as $\lambda$ goes to infinity, the trajectories of the reduced system when $r(t)$ and $d(t)$ vary slowly stay increasingly close to the locally unique equilibrium output computed assuming $r$ and $d$ constant. Our main result relies on finding conditions under which the above properties are sufficient to ensure that as $\epsilon \rightarrow 0$ and $\lambda \rightarrow \infty$, the output $y(t)$ is close to the reference, $r(t)$, no matter what the disturbance, $d(t)$, does.

The plant subsystem, $P$, takes as its inputs $\bar{u}$ and $d$. It has two outputs, $y$ and $\bar{x}$.

$$
P=\left\{\begin{array}{l}
\dot{\bar{x}}=\bar{f}(\bar{x}, \bar{u}, d) \\
y=h(\bar{x})
\end{array}\right.
$$

The controller subsystem, $C(\epsilon, \lambda)$, takes as its inputs $\bar{x}$ and $r$, and has as its output $\bar{u}$.

$$
C(\epsilon, \lambda)=\left\{\begin{aligned}
\epsilon \dot{\bar{z}} & =\bar{g}(\bar{x}, \bar{z}, r, \lambda) \\
\bar{u} & =h_{c}(\bar{z})
\end{aligned}\right.
$$

Together, (1) and (2) result in the closed loop system

$$
\begin{aligned}
\dot{\bar{x}} & =\bar{f}\left(\bar{x}, h_{c}(\bar{z}), u\right), \\
\epsilon \dot{\bar{z}} & =\bar{g}(\bar{x}, \bar{z}, u, \lambda), \\
y & =h(\bar{x}) .
\end{aligned}
$$

Where $u=(r, d)$. Let $q(u, \lambda)=\left(q_{1}(u, \lambda), q_{2}(u, \lambda)\right)$ be a locally isolated solution of $0=\bar{f}\left(q_{1}(u, \lambda), h_{c}\left(q_{2}(u, \lambda)\right), d\right)$, $0=\bar{g}\left(q_{1}(u, \lambda), q_{2}(u, \lambda), r, \lambda\right)$, i.e. the equilibrium value of $(\bar{x}, \bar{z})$ for constant $u$. We assume that $u(t)$ takes values in a compact set, $\mathcal{U}$, and that the domain of (3) is $\overline{\mathcal{D}}$.

We consider a design approach that guarantees $y(t)$ is close to $r(t)$ independent of $d(t)$ for a set of slowly varying references and disturbances, as well as initial conditions that do not depend on the parameter $\lambda$, in the limit as $\lambda \rightarrow \infty$ and $\epsilon \rightarrow 0$. We formalize this specification, which we call asymptotic robust reference tracking.

Definition 2.1: A system of the form (3) has the asymptotic robust reference tracking property if there exists functions, $\epsilon^{*}(\lambda)$ and $\alpha(\lambda), p>0$, and set $\mathcal{U} \subset \mathbb{R}^{p+q}$ independent of $\lambda, \lambda^{*} \in \mathbb{R}$, and an $\eta>0$ such that $\forall \lambda \geq \lambda^{*}$, if $\epsilon<\epsilon^{*}(\lambda)$, we have that for all $u=(r, d) \in \mathcal{U},\|(\dot{r}, \dot{d})\|<\eta$, and $(\bar{x}(0), \bar{z}(0)) \in B_{p}\left(\left(q_{1}(u(0), \lambda), q_{2}(u(0), \lambda)\right)\right) \subset \overline{\mathcal{D}}^{1}$,

$$
\lim _{\substack{t \rightarrow \infty \\ \text { Rark 2.1: For convenience we will consider the }}}\|y(t)-r(t)\| \leq \alpha(\lambda) \text {, and } \lim _{\lambda \rightarrow \infty} \alpha(\lambda)=0 .
$$

Remark 2.1: For convenience we will consider the set $\mathcal{P}=B_{p}((0,0))$, so that the condition $(\bar{x}(0), \bar{z}(0)) \in$ $B_{p}\left(\left(q_{1}(u(0), \lambda), q_{2}(u(0), \lambda)\right)\right)$ in Definition 2.1 can be replaced with $\left(\bar{x}(0)-q_{1}(u(0), \lambda), \bar{z}(0)-q_{2}(u(0), \lambda)\right) \in \mathcal{P}$.

Remark 2.2: Definition 2.1 implies that for constant reference, $r$, and disturbance, $d$, we have $\forall u \in$ $\mathcal{U}, \lim _{\lambda \rightarrow \infty} h\left(q_{1}(u, \lambda)\right)=r$. This means that the equilibrium value of the output is asymptotically insensitive to the disturbance, and asymptotically approaching the reference.

Remark 2.3: Definition 2.1 essentially requires that for all $\lambda>\lambda^{*}$ we can apply singular perturbation to system (3) in $\epsilon$, in such a way that a) the reduced system has the asymptotic reference robust tracking property and $b$ ) the singular perturbation analysis holds on $t \in[0, \infty)$.

Based on Remark 2.2 we can search for systems of the form (3) that have the asymptotic reference tracking property in two steps. First, we require that the system has the property described in Remark 2.2. We then ensure that $\left.\lim _{t \rightarrow \infty} \| \bar{x}(t)-q_{1}(u(t), \lambda), \lambda\right) \|$ approaches zero as $\lambda \rightarrow \infty$ for all $r(t), d(t)$ changing sufficiently slowly, and all initial conditions in a set that does not shrink to zero volume as $\lambda \rightarrow \infty$.

The problem studied in this work is determining classes of systems of the form (3) which satisfy Definition 2.1. Before proceeding we note that asymptotic robust tracking cannot be guaranteed by analysis based solely on linearization.

Example 2.1: Consider the following system which fits into the form of (3).

$$
\begin{aligned}
\dot{\bar{x}} & =\bar{z}-\bar{x}, \\
\epsilon \dot{\bar{z}} & =-\lambda \bar{x}-\bar{z}+\lambda^{2} \bar{z}^{3} .
\end{aligned}
$$

${ }^{1} B_{p}((x, z))$ denotes the open ball of radius $p$ centered at $(x, z)$. 
Note that while no $r$ or $d$ appears in our dynamics, i.e. $q(r, d, \lambda)=0$, we let $\mathcal{U}=\{(0,0)\}$ and investigate whether (4) has the asymptotic robust reference tracking property. We show that this is not the case by showing that trajectories starting at $\bar{x}(0)=\sqrt{(\lambda+1) / \lambda^{2}}=: \bar{x}_{0}, \bar{z}(0)=$ $\left.\sqrt{(\lambda+1) / \lambda^{2}}+\eta\right)=: \bar{z}_{0}, \eta>0$, diverge to infinity. First, we show that $S=\left\{(\bar{x}, \bar{z}) \mid \bar{x} \geq \sqrt{(\lambda+1) / \lambda^{2}}, \bar{z} \geq \bar{x}\right\}$ is positively invariant. To do this, observe that in $S \dot{\bar{x}} \geq 0$, and hence $\bar{x}$ cannot decrease below $\sqrt{(\lambda+1) / \lambda^{2}}$. Observe also that $\forall(\bar{x}, \bar{z}) \in S, \bar{x} \leq-\bar{z} / \lambda+\lambda \bar{z}^{3}$, and so by (4b), $\dot{\bar{z}} \geq 0$ in $S$. This combined with (4a) means that trajectories cannot cross the boundary of $S$ defined by $\bar{x}=\bar{z}$. Therefore, along the trajectory starting at $\left(\bar{x}_{0}, \bar{z}_{0}\right), \dot{\bar{z}}>\lambda^{2} \eta^{3} / \epsilon$, and hence $\lim _{t \rightarrow \infty} \bar{z}(t)=\infty$. Therefore, there does not exist an open ball, $B_{r}$, about $(0,0)$ such that $B_{r}$ is contained for all $\lambda$ within the region of attraction of $(0,0)$. Hence Definition 2.1 cannot be satisfied. This cannot be deduced from the the linearization of (4) about $(0,0)$, which is $\dot{\bar{x}}=\bar{z}-\bar{x}$, $\epsilon \dot{\bar{z}}=-\lambda \bar{x}-\bar{z}$. If we set $\epsilon(\lambda)=1 / \lambda^{2}$, the eigenvalues of the linearization all have negative real part and are bounded away from the imaginary axis uniformly in $\lambda$. Therefore, the linearized system does not indicates that the region of attraction shrinks as $\lambda \rightarrow \infty$.

To deal with the issue shown by Example 2.1, in the next section we present sufficient conditions for asymptotic robust reference tracking.

\section{MAIN RESULT}

In this section, we present the main result of this work, conditions under which asymptotic robust reference tracking is achieved. To do this, we use Lyapunov analysis, which necessitates construction of a Lyapunov function for system (3). For sufficiently small $\epsilon$, this is possible by taking a convex combination of Lyapunov functions for the boundary layer and reduced systems [25]. This procedure, which under additional technical conditions results in a Lyapunov function for (3) that proves a bound on trajectories when the exogenous inputs are slowly varying, is detailed in Lemma 3.1. The bound that is shown goes to zero as $\lambda$ goes to infinity under additional assumptions as detailed in Lemma 3.2. We then specialize Lemma 3.1 to cases where the slow dynamics are one dimensional, we have a Lyapunov function for the boundary layer, and asymptotic robust tracking is assured by the reduced system's behavior.

Considering (3), we now prove a bound on system trajectories relative to the equilibrium point that holds when we have Lyapunov functions for the reduced and boundary layer systems. Let $u=(r, d)$ be the exogenous inputs consisting of the reference, $r$, and the disturbance, $d$. Here $r \in \mathbb{R}^{p}$, $d \in \mathbb{R}^{q}$ are time varying. Additionally, $\epsilon>0$, and $\lambda \in \mathbb{R}_{+}$. We now develop some basic notions of singular perturbation analysis that we will need to state and prove Lemma 3.1. Let $\left(q_{1}(u, \lambda), q_{2}(u, \lambda)\right)$ be a locally isolated solution to

$$
\begin{aligned}
& 0=\bar{f}\left(q_{1}(u, \lambda), h_{c}\left(q_{2}(u, \lambda)\right), d\right), \\
& 0=\bar{g}\left(q_{1}(u, \lambda), q_{2}(u, \lambda), r, \lambda\right),
\end{aligned}
$$

We assume that $\left(q_{1}(u, \lambda), q_{2}(u, \lambda)\right)$ is differentiable. Letting $x=\bar{x}-q_{1}(u, \lambda)$ and $z=\bar{z}-q_{2}(u, \lambda)$ the dynamics of (3) in $(x, z)$ coordinates are

$$
\begin{aligned}
\dot{x} & =f(x, z, u)-\frac{\partial q_{1}}{\partial u} \dot{u}, \\
\epsilon \dot{z} & =g(x, z, u, \lambda)-\epsilon \frac{\partial q_{2}}{\partial u} \dot{u},
\end{aligned}
$$

where $f(x, z, u)=\bar{f}\left(x+q_{1}(u, \lambda), h_{c}\left(z+q_{2}(u, \lambda)\right), u\right)$ and $g(x, z, u, \lambda)=\bar{g}\left(x+q_{1}(u, \lambda), z+q_{2}(u, \lambda), u, \lambda\right)$. Let $H(x, u, \lambda)$ be an isolated solution to $0=$ $g(x, H(x, u, \lambda), u, \lambda)$ and define $y_{b}=z-H(x, u, \lambda)$. Let

$$
\mathcal{D}(u, \lambda)=\left\{(x, z) \mid\left(x+q_{1}(u, \lambda), z+q_{2}(u, \lambda)\right) \in \overline{\mathcal{D}}\right\},
$$

which is the domain of (6). The reduced system is

$$
\dot{x}_{r}=f\left(x_{r}, y_{b}+H\left(x_{r}, u, \lambda\right), u\right) .
$$

Let $\tau=t / \epsilon$. The boundary layer equation is

$$
\frac{d y_{b}}{d \tau}=g\left(x, y_{b}+H(x, u, \lambda), u, \lambda\right)
$$

Assumption 3.1: There exists $B>0, \lambda^{*} \in \mathbb{R}$ such that

$$
\forall \bar{x} \in \overline{\mathcal{D}}, \forall u \in \mathcal{U}, \forall \lambda \geq \lambda^{*},\left\|\frac{\partial q_{1}}{\partial u}\right\|,\left\|\frac{\partial q_{2}}{\partial u}\right\| \leq B .
$$

Assumption 3.2: There exists $\lambda^{*} \in \mathbb{R}$ such that for all $\lambda \geq \lambda^{*}$ there exists $L(\lambda)>0$ which satisfies

$$
\forall u \in \mathcal{U}, \forall \lambda \geq \lambda^{*}, \forall x \in \mathcal{D}(u, \lambda),\left\|\frac{\partial H}{\partial x}\right\| \leq L(\lambda),
$$

where $L(\lambda)$ is bounded away from zero, and furthermore, there exists $L^{\prime}>0$ independent of $\lambda$ and $u$ such that

$$
\forall u \in \mathcal{U}, \forall \lambda \geq \lambda^{*}, \forall x \in \mathcal{D}(u, \lambda),\left\|\frac{\partial H}{\partial u}\right\| \leq L^{\prime} .
$$

Assumption 3.3: Assume $\exists V: \mathbb{R}^{m} \rightarrow \mathbb{R}$, and $v_{0}>0$, both independent of $\lambda \geq \lambda^{*}$, and $R: \mathbb{R} \rightarrow \mathbb{R}$ such that $\forall \lambda>\lambda^{*}, R(\lambda)>0$ which satisfy for all constant $u \in \mathcal{U}$,

$$
\begin{aligned}
& \forall x \in\left\{x \in \operatorname{proj}_{x}(D(u, \lambda)) \mid V(x) \leq v_{0}\right\} \\
& b_{1}\|x\|_{2}^{2 a_{v}} \leq V(x) \leq b_{2}\|x\|_{2}^{2 a_{v}},
\end{aligned}
$$

where $\operatorname{proj}_{x}(T)$ is the projection of set $T$ onto the $x$ coordinates, and for all constant $u \in \mathcal{U}$,

$$
\begin{array}{r}
\forall x \in\left\{x \in \operatorname{proj}_{x}(D(u, \lambda)) \mid R(\lambda)<\|x\|_{2}^{a_{v}}, V(x) \leq v_{0}\right\} \\
\frac{\partial V}{\partial x} f(x, H(x, u, \lambda), u) \leq-b_{3}^{\prime}\|x\|_{2}^{a_{v}},
\end{array}
$$

with $b_{1}, b_{2}, b_{3}^{\prime}$, and furthermore, $\exists k_{1}(\lambda)>0$ such that

$$
\forall u \in \mathcal{U},\left\|\frac{\partial V}{\partial x}\right\| \leq k_{1}(\lambda)\|x\|_{2}^{a_{v}}
$$

Assumption 3.4: Assume there exists a Lyapunov function, $W\left(y_{b}\right)$, for system (8), and constants $c_{1}, c_{2}, c_{3}$, all independent of $\lambda$, which satisfy for constant $u \in \mathcal{U}, \forall y_{b} \in$ $\left\{y_{b}^{\prime} \mid \exists x\right.$ s.t. $\left.y_{b}^{\prime}+H(x, u, \lambda) \in \operatorname{proj}_{z} \mathcal{D}\right\}$,

$$
c_{1}\left\|y_{b}\right\|_{2}^{2 a_{w}} \leq W\left(y_{b}\right) \leq c_{2}\left\|y_{b}\right\|_{2}^{2 a_{w}}
$$




$$
\begin{aligned}
\forall u & \in \mathcal{U}, \forall V(x) \leq v_{0}, \\
& \frac{\partial W}{\partial y_{b}} g\left(x, y_{b}+H(x, u, \lambda), u, \lambda\right) \leq-c_{3}\left\|y_{b}\right\|_{2}^{2 a_{w}},
\end{aligned}
$$

with $c_{1}, c_{2}, c_{3}>0$, and additionally, $\exists k_{4}(\lambda)>0$ such that

$$
\forall u \in \mathcal{U},\left\|\frac{\partial W}{\partial y_{b}}\right\| \leq k_{4}(\lambda)\left\|y_{b}\right\|_{2}^{a_{w}} .
$$

Assumption 3.5: Assume that on the set $S^{*}=$ $\left\{\left(x, y_{b}\right) \mid \frac{V(x)}{v_{0}}+\frac{W\left(y_{b}\right)}{w_{0}(\lambda)} \leq 1\right\}$, where $V, v_{0}, W$ and $w_{0}$ are as in Assumptions 3.3 and 3.4, we have with $u(t)$ constant the following conditions $\forall u \in \mathcal{U}$ :

$$
\|f(x, H(x, u, \lambda), u)\| \leq k_{2}\|x\|_{2}^{a_{v}},
$$

$$
\left\|f\left(x, y_{b}+H(x, u, \lambda), u\right)-f(x, H(x, u, \lambda), u)\right\| \leq k_{3}\left\|y_{b}\right\|_{2}^{a_{w}},
$$

where $k_{2}(\lambda), k_{3}(\lambda)$ are positive functions of $\lambda$.

We are now ready to state our first lemma.

Lemma 3.1: Consider the system (6). Suppose that Assumptions 3.1, 3.2, 3.3, 3.4, and 3.5 are satisfied. Define

$$
\begin{aligned}
& \hat{\mathcal{P}}=\left\{(x, z) \mid \frac{b_{2}}{v_{0}}\|x\|_{2}^{2 a_{v}}+\frac{c_{2}}{w_{0}(\lambda)}\|z\|_{2}^{2 a_{w}}\right. \\
& \left.+\frac{2 c_{2} L(\lambda)^{a_{w}}}{w_{0}(\lambda)}\|z\|^{a_{w}}\|x\|^{a_{w}}+\frac{c_{2} L(\lambda)^{2 a_{w}}}{w_{0}(\lambda)}\|x\|^{2 a_{w}} \leq 1\right\} .
\end{aligned}
$$

Under these conditions we have that if $\forall t \geq 0, u(t) \in \mathcal{U}$ and $\|\dot{u}(t)\|<\frac{b_{3}^{\prime}}{2 k_{1} B}$, then $\forall \lambda>0, \bar{K}(\lambda)>0, \exists \epsilon^{* * *}>0$ such that $\forall(x(0), z(0)) \in \hat{\mathcal{P}}, 0<\epsilon<\epsilon^{* * *}$,

$$
\limsup _{t \rightarrow \infty}\|x(t)\|_{2} \leq\left(\frac{b_{1}(1-D) R^{2}(\lambda)+c_{1} D \bar{K}^{2}(\lambda)}{b_{1}(1-D)}\right)^{\frac{1}{2 a_{v}}},
$$

and

$$
\begin{aligned}
\limsup _{t \rightarrow \infty}\|z(t)-H(t, x(t))\|_{2} \\
\leq\left(\frac{b_{1}(1-D) R^{2}(\lambda)+c_{1} D \bar{K}^{2}(\lambda)}{c_{1} D}\right)^{\frac{1}{2 a_{w}}},
\end{aligned}
$$

where $D=v_{0} /\left(v_{0}+w_{0}\right)$.

Proof: See Appendix A.

Remark 3.1: The right-hand sides of (18) and (19) do not depend on $\dot{u}$. In fact, the conditions of Lemma 3.1 do not guarantee that $\|x(t)\|_{2}$ or $\|z(t)-H(t, x(t))\|_{2}$ tend to zero as $\dot{u}$ goes to zero. This is due to us only knowing about the behavior of the reduced system for $\|x\|>R(\lambda)$.

Remark 3.2: To use Lemma 3.1 to show that a system of the form (3) has the asymptotic robust reference tracking property of Definition 2.1 , we must have that $\lim _{\lambda \rightarrow \infty} R(\lambda)=0$. We then pick $\bar{K}(\lambda)$ such that $\lim _{\lambda \rightarrow \infty} \bar{K}(\lambda)=0$, e.g. $\bar{K}(\lambda)=1 / \lambda$, and pick $w_{0}(\lambda)=$ $\Omega\left(L^{2}(\lambda)\right)^{2}$, e.g. $w_{0}(\lambda)=\bar{k} L^{2}(\lambda)$ for some $\bar{k}>0$. These conditions ensure that $\exists \mathcal{P} \subseteq \hat{\mathcal{P}}$ with nonzero volume that is independent of $\lambda$, and that the bound on the right-hand side of (18) go to zero as $\lambda$ goes to infinity. The bound on the right-hand side of (19) may or may not go to zero as $\lambda$ goes to infinity, depending on how fast $R^{2}(\lambda)$ goes to zero

${ }^{2}$ i.e. $\exists k>0, \lambda * \in \mathbb{R}$ such that $\forall \lambda>\lambda^{*}, w_{0}(\lambda) \geq k L^{2}(\lambda)$. compared to $1 / w_{0}(\lambda)$, however it is not necessary that the right-hand side of (19) go to zero as $\lambda$ goes to infinity for Definition 2.1 to hold.

The following assumption will be needed in order to use Lemma 3.1 to prove asymptotic robust reference tracking.

Assumption 3.6: Consider system (3). Assume also that for the output $y=h(\bar{x})$, where $h$ is assumed to be Lipschitz continuous on $\overline{\mathcal{D}}$, we have for constant $u=(r, d)$,

$$
\forall u \in \mathcal{U}, \lim _{\lambda \rightarrow \infty} h\left(q_{1}(u, \lambda)\right)=r .
$$

We now give a lemma that shows that under suitable additional conditions, Lemma 3.1 can be used to guarantee that system (3) has the asymptotic robust reference tracking property of Definition (2.1).

Lemma 3.2: Consider system (3). Suppose that Assumptions 3.1, 3.2, 3.3, 3.4, and 3.5 are satisfied, where Assumption 3.3 is satisfied with an $R(\lambda)$ such that $\lim _{\lambda \rightarrow \infty} R(\lambda)=$ 0 , and Assumption 3.4 is satisfied for all $w_{0}>0$ with some $c_{1}, c_{2}, c_{3}$ that are independent of $w_{0}$. Assume also that (3) satisfies Assumption 3.6. Then, system (3) has the asymptotic robust reference tracking property of Definition 2.1.

Remark 3.3: The assumption that $\lim _{\lambda \rightarrow \infty} R(\lambda)=0$ requires that we find a $V(x)$ with particular properties stronger than Assumption 3.3.

Proof: Since Assumptions 3.1, 3.2, 3.3, 3.4, and 3.5 are satisfied, we can apply Lemma 3.1. Due to our additional assumptions, we are allowed to choose $w_{0}(\lambda)=L(\lambda)^{2 a_{w}}$. Then, $\hat{\mathcal{P}}$ in the conclusion of Lemma 3.1 is

$$
\begin{aligned}
\hat{\mathcal{P}}=\{(x, z) \mid & \mid \frac{b_{2}}{v_{0}}\|x\|_{2}^{2 a_{v}}+\frac{c_{2}}{L(\lambda)^{2 a_{w}}}\|z\|_{2}^{2 a_{w}} \\
& \left.+\frac{2 c_{2}}{L(\lambda)^{a_{w}}}\|z\|^{a_{w}}\|x\|^{a_{w}}+c_{2}\|x\|^{2 a_{w}} \leq 1\right\} .
\end{aligned}
$$

Due to Assumption 3.2 requiring that $L(\lambda)$ be bounded away from zero, we have that $L_{\text {min }}=\inf _{\lambda \geq \lambda^{*}} L(\lambda)>0$. Therefore, we have that $\forall \lambda \geq \lambda^{*}, \mathcal{P} \subseteq \hat{\mathcal{P}}$, where

$$
\begin{aligned}
\mathcal{P}=\{(x, z) \mid & \frac{b_{2}}{v_{0}}\|x\|_{2}^{2 a_{v}}+\frac{c_{2}}{L_{m i n}^{2 a_{w}}}\|z\|_{2}^{2 a_{w}} \\
& \left.+\frac{2 c_{2}}{L_{m i n}^{a_{w}}}\|z\|^{a_{w}}\|x\|^{a_{w}}+c_{2}\|x\|^{2 a_{w}} \leq 1\right\} .
\end{aligned}
$$

Observe that $\mathcal{P}$ is independent of $\lambda$. We then pick $\bar{K}=$ $1 / \lambda$, which $\forall(x(0), z(0)) \in \mathcal{P}, 0<\epsilon<\epsilon^{* * *}$ results in the following bound in $(x, z)$ coordinates:

$$
\limsup _{t \rightarrow \infty}\|x(t)\|_{2} \leq\left(\frac{b_{1}(1-D) R^{2}(\lambda)+c_{1} D / \lambda^{2}}{b_{1}(1-D)}\right)^{\frac{1}{2 a_{v}}},
$$

where $D=v_{0} /\left(v_{0}+L(\lambda)^{2}\right)$. From (20) and compactness of $\mathcal{U}$, we have that $\exists K_{c}(\lambda)>0$ and $\lambda^{*}$ such that

$$
\forall(r, d) \in \mathcal{U}, \forall \lambda>\lambda^{*},\left\|h\left(q_{1}(u, \lambda)\right)-r\right\|_{2} \leq K_{c}(\lambda),
$$

where $\lim _{\lambda \rightarrow \infty} K_{c}(\lambda)=0$. Now consider $y(t)$. We have that $y(t)=h\left(x(t)+q_{1}(u(t), \lambda)\right)$. From (22) and because $h$ is 
Lipschitz we have $\forall(x(0), z(0)) \in \mathcal{P}, 0<\epsilon<\epsilon^{* * *}, \forall t \geq$ $0, u(t) \in \mathcal{U}, \lambda>\lambda^{*}$,

$$
\begin{array}{r}
\left\|h\left(x(t)+q_{1}(u(t), \lambda)\right)-r(t)\right\|_{2} \\
\leq\left\|h\left(x(t)+q_{1}(u(t), \lambda)\right)-h\left(q_{1}(u(t), \lambda)\right)\right\|_{2} \\
+\left\|h\left(q_{1}(u(t), \lambda)\right)-r(t)\right\|_{2} \\
\quad \leq K_{h}\|x(t)\|_{2}+K_{c}(\lambda),
\end{array}
$$

where $K_{h}$ is the Lipschitz constant of $h$. Taking limit superior of both sides of this expression we obtain, via (21), $\forall\left(\bar{x}(0)-q_{1}(u, \lambda), \bar{z}(0)-q_{2}(u, \lambda)\right) \in \mathcal{P}, 0<\epsilon<\epsilon^{* * *}, \forall t \geq$ $0, u(t) \in \mathcal{U}, \lambda>\lambda^{*}$

$$
\begin{aligned}
& \limsup _{t \rightarrow \infty}\left\|h\left(x(t)+q_{1}(u(t), \lambda)\right)-r(t)\right\|_{2} \\
& \quad \leq K_{h}\left(\frac{b_{1}(1-D) R^{2}(\lambda)+c_{1} D / \lambda^{2}}{b_{1}(1-D)}\right)^{\frac{1}{2 a_{v}}}+K_{c}(\lambda) .
\end{aligned}
$$

Since the right-hand side of the above expression tends to zero as $\lambda \rightarrow \infty$, we have shown that system (3) has the asymptotic disturbance rejection property.

We next present a lemma which uses Lemma 3.1 to find simple conditions that guarantee asymptotically tracking performance for systems where the slow dynamics are one dimensional. We first state and prove the following Lemma, which we will use to analyze the reduced system.

Lemma 3.3: Consider a continuous function, $\hat{f}(x, u, \lambda)$ : $\mathbb{R} \times \mathbb{R} \times \mathbb{R} \rightarrow \mathbb{R}$, and suppose that on some domain $|x| \leq v_{0}$, $\lambda \geq \lambda_{0}$ we have some $\beta>0$ such that $\forall u \in \mathcal{U}, \hat{f}(0, u, \lambda)=$ 0 and

1) $\hat{f}(x, u, \lambda)$ is strict monotonic decreasing,

2) $|\hat{f}(x, u, \lambda)|$ is strict monotonic increasing with respect to $\lambda$,

3) $\lim _{\lambda \rightarrow \infty} \hat{f}(x, u, \lambda) \begin{cases}\geq \beta, & \forall x<0, \\ \leq-\beta, & \forall x>0,\end{cases}$

Then, there are $\lambda^{*} \in \mathbb{R}, \beta>\epsilon>0$ such that $\forall \lambda>\lambda^{*}$, $\exists R(\lambda)>0$ such that $\forall|x|>R(\lambda)$,

$$
|\hat{f}(x, u, \lambda)|>\beta-\epsilon=\beta^{\prime} \text { and } \lim _{\lambda \rightarrow \infty} R(\lambda)=0
$$

Proof: See Appendix B.

Using Lemma 3.3 we are now ready to study systems of the form (3) when the slow dynamics are one dimensional, i.e., $\bar{x} \in \mathbb{R}$. The following theorem is the main result of this paper, which gives conditions under which systems of the form (3) with $\bar{x} \in \mathbb{R}$ have the asymptotic robust reference tracking property.

Theorem 3.1: Consider system (3) and suppose that $\bar{x} \in$ $\mathbb{R}$. Suppose that Assumptions 3.1, 3.2, and 3.4, are satisfied. Assume that the conditions of Lemma 3.3 are satisfied by $\hat{f}(x, u, \lambda)=f(x, H(x), u, \lambda)$, i.e., (7), and that with $y=h(\bar{x})$, system (3) satisfies Assumption 3.6. Then, system (3) has the robust reference tracking property according to Definition 2.1.

Proof: We will apply Lemma 3.2 by using Lemma 3.3 to show the existence of a Lyapunov function for the reduced system that satisfies the conditions of Assumption 3.3.
Let $V(x)=x^{2}$. Then along trajectories of the reduced system, we have from Lemma 3.3 that for sufficiently large $\lambda, \forall u \in \mathcal{U}, \forall R(\lambda)<|x| \leq v_{0}$,

$$
\frac{\partial V}{\partial x} f(x, H(x), u, \lambda)=2 x f(x, H(x), u, \lambda) \leq-2 \beta^{\prime}|x|,
$$

where $\beta^{\prime}$ is as in (23). Thus, with $a_{v}=a_{w}=1$ and $b_{1}=$ $b_{2}=1$, and $b_{3}^{\prime}=2 \beta^{\prime}$, equations eqs. (9) and (10) are satisfied with our choice of $V(x)$, and therefore Assumption 3.3 is satisfied for system (3) with $\lim _{\lambda \rightarrow \infty} R(\lambda)=0$.

We now need to ensure that Assumption 3.5 is satisfied by system (3) and our choice of $V(x)$. Observe that (11) is satisfied with $k_{1}=2$. Since $f$ and $H$ are Lipschitz, we have that $\exists k_{2}$ such that (15) is satisfied, and $\exists k_{3}$ such that (16) is satisfied, and hence Assumption 3.5 is satisfied by system (3) with $V(x)=x^{2}$. Therefore we can apply Lemma 3.2 to obtain the desired result.

\section{STRUCTURAL RESULTS}

To identify systems of the form (3) that satisfy Definition 2.1 , we now introduce a class of systems where the plant, $P$, and controller, $C$, each have a special structure. We give conditions under which systems of this class have the asymptotic robust reference tracking property of Definition 2.1. We consider the case where $P$ is given by $\dot{\bar{x}}=\gamma(\bar{u}, d)-\delta \bar{x}$, where $\bar{x} \in \mathbb{R}$, and $\delta>0$, and $\gamma: \mathbb{R} \times \mathbb{R} \rightarrow \mathbb{R}$. This results in $f$ in (3) being given by $f(\bar{x}, \bar{u}, d)=\gamma(\bar{u}, d)-\delta \bar{x}$. We consider the following dynamics for $C$, which are inspired by antithetic feedback control [7], [10], [13]:

$$
\begin{aligned}
& \epsilon \dot{\bar{z}}_{1}=\lambda\left(g_{1}(r)+g_{2}\left(\bar{z}_{1}, \bar{z}_{2}\right)\right)-\bar{z}_{1}, \\
& \epsilon \dot{\bar{z}}_{2}=\lambda\left(g_{3}(\bar{x})+g_{2}\left(\bar{z}_{1}, \bar{z}_{2}\right)\right)-\bar{z}_{2},
\end{aligned}
$$

where $\bar{z}_{1}, \bar{z}_{2} \in \mathbb{R}$. i.e. $g$ in (3) is given by

$$
g(\bar{x}, \bar{z}, r, \lambda)=\left[\begin{array}{l}
\lambda\left(g_{1}(r)+g_{2}\left(\bar{z}_{1}, \bar{z}_{2}\right)\right)-\bar{z}_{1} \\
\lambda\left(g_{3}(\bar{x})+g_{2}\left(\bar{z}_{1}, \bar{z}_{2}\right)\right)-\bar{z}_{2},
\end{array}\right]
$$

and $\bar{u}=h_{c}(\bar{z})=\bar{z}_{1}$. This leads to the following closed loop system:

$$
\begin{aligned}
\dot{\bar{x}} & =\gamma\left(\bar{z}_{1}, d\right)-\delta \bar{x}, \\
\epsilon \dot{\bar{z}}_{1} & =\lambda\left(g_{1}(r)+g_{2}\left(\bar{z}_{1}, \bar{z}_{2}\right)\right)-\bar{z}_{1}, \\
\epsilon \dot{\bar{z}}_{2} & =\lambda\left(g_{3}(\bar{x})+g_{2}\left(\bar{z}_{1}, \bar{z}_{2}\right)\right)-\bar{z}_{2} .
\end{aligned}
$$

We will show that under mild technical conditions, (25) has the asymptotic robust reference tracking property. Specifically, we give conditions on $g_{1}, g_{2}, g_{3}, \gamma$ such that (25) has the asymptotic robust reference tracking property. We make the following assumptions:

Assumption 4.1: For (25) we assume that $\bar{z}_{1}, \bar{z}_{2} \in \mathbb{R}$, and on the positive orthant $g_{1}, g_{2}, g_{3}$ are continuously differentiable, $g_{2}<0, g_{1}, g_{3}>0, \partial g_{2} / \partial \bar{z}_{1}<0, \partial g_{2} / \partial \bar{z}_{2}<0$, $\partial g_{3} / \partial \bar{x}>0$, and $r, \epsilon, \lambda>0$. We assume that for each value of $\bar{x}>0$, there is a unique solution to (24), $\left(z_{1}^{*}, z_{2}^{*}\right)$ in the non-negative quadrant and that $g_{1}$ is invertible on $\mathbb{R}_{\geq 0}$. We also assume that $\bar{x} \in \mathbb{R}, \delta>0$, and on the positive orthant $\gamma>0$ and $\partial \gamma / \partial \bar{z}_{1}>0$ exists and is continuous, and that $g_{2}\left(0, \bar{z}_{2}\right)=g_{2}\left(\bar{z}_{1}, 0\right)=0$. 
Here the last condition serves to enforce positive invariance of the positive quadrant.

Corollary 4.1: Consider (25) under Assumption 4.1. Then we have that (25) satisfies Definition 2.1 with some output $y=h(\bar{x})$.

Proof: Our proof proceeds by applying Lemma 3.1 to (25). We first examine the behavior of the equilibrium point as $\lambda$ goes to infinity. The solution to

$$
\begin{aligned}
& 0=\lambda\left(g_{1}(r)+g_{2}\left(\bar{z}_{1}, \bar{z}_{2}\right)\right)-\bar{z}_{1}, \\
& 0=\lambda\left(g_{3}(\bar{x})+g_{2}\left(\bar{z}_{1}, \bar{z}_{2}\right)\right)-\bar{z}_{2},
\end{aligned}
$$

approaches that of

$$
0=g_{1}(r)+g_{2}\left(\bar{z}_{1}, \bar{z}_{2}\right), \quad 0=g_{3}(\bar{x})+g_{2}\left(\bar{z}_{1}, \bar{z}_{2}\right),
$$

as $\lambda \rightarrow \infty$. Equation (27) has a solution if and only if $g_{1}(r)=g_{3}(\bar{x})$. Therefore, $\lim _{\lambda \rightarrow \infty} g_{1}^{-1}\left(g_{3}\left(x^{*}(u, d, \lambda)\right)=r\right.$. This establishes that assumption 3.6 is satisfied. Now observe that using the implicit function theorem on (26) we have $\frac{\partial z^{*}}{\partial \bar{x}}=-M^{-1}\left[\begin{array}{ll}0 & \lambda \frac{\partial g_{3}}{\bar{x}}\end{array}\right]^{T}$, where $m_{11}=\lambda \frac{\partial g_{2}}{\partial \bar{z}_{1}}-1, m_{12}=$ $\lambda \frac{\partial g_{2}}{\partial \bar{z}_{2}}, m_{21}=\lambda \frac{\partial g_{2}}{\partial \bar{z}_{1}}$, and $m_{22}=\lambda \frac{\partial g_{2}}{\partial \bar{z}_{2}}-1$. By the Gershgorin Circle Theorem [26], $\operatorname{det} M>0$, and so $\partial z_{1}^{*} / \partial \bar{x}<0$. This ensures that the reduced system, where $\epsilon=0$ is globally asymptotically stable on $\mathbb{R}_{>0}$. We now consider a Lyapunov function for the boundary layer system. Consider fixed values of $r$ and $\bar{x}$. Let $W=\left\|\bar{z}-z^{*}\right\|_{1}^{2}$ [27]. Note that although $W$ is not differentiable, its forward derivative along $\dot{\bar{z}}$ is always defined. We consider the derivative of $W$ in four different regions that form a partition of $\bar{z}_{1}, \bar{z}_{2} \geq 0$.

1) $\bar{z}_{1}>z_{1}^{*}, \bar{z}_{2}>z_{2}^{*}$ : In this region, we have that $\dot{\bar{z}}_{1}<$ $-\left(\bar{z}_{1}-z_{1}^{*}\right)$ and $\dot{\bar{z}}_{2}<-\left(\bar{z}_{2}-z_{2}^{*}\right)$ and hence, $\dot{W}=$ $2\left\|\bar{z}-z^{*}\right\|\left(\dot{\bar{z}}_{1}+\dot{\bar{z}}_{2}\right)<2\left\|\bar{z}-z^{*}\right\|^{2}=2 W$.

2) $\bar{z}_{1}<z_{1}^{*}, \bar{z}_{2}>z_{2}^{*}$ : In this region, we have that $\dot{W}=$ $2\left\|\bar{z}-z^{*}\right\|\left(-\dot{\bar{z}}_{1}+\dot{\bar{z}}_{2}\right)<2\left\|\bar{z}-z^{*}\right\|^{2}=2 W$.

3) $\bar{z}_{1}<z_{1}^{*}, \bar{z}_{2}<z_{2}^{*}$ : First, consider the fact that since $\bar{z}_{1}, \bar{z}_{2} \geq 0$ is positively invariant, we only need $\dot{W}<0$ on that set. Now, observe that because $\partial g_{2} / \partial \bar{z}_{1}<0$ and $\partial g_{2} / \partial \bar{z}_{2}<0$, we have that $\forall \bar{z}_{1}<$ $z_{1}^{*}, \bar{z}_{2}<z_{2}^{*}, \quad \dot{\bar{z}}_{1}, \dot{\bar{z}}_{2}>0$. Since in this quadrant, $\dot{W}=2\left\|\bar{z}-z^{*}\right\|\left(-\dot{\bar{z}}_{1}-\dot{\bar{z}}_{2}\right)<0$.

4) $\bar{z}_{1}>z_{1}^{*}, \bar{z}_{2}<z_{2}^{*}$ : In this region, we have that $\dot{W}=$ $2\left\|\bar{z}-z^{*}\right\|\left(\dot{\bar{z}}_{1}-\dot{\bar{z}}_{2}\right)<2\left\|\bar{z}-z^{*}\right\|^{2}=2 W$.

Therefore, $\forall w_{0}>0$, we have (12)-(14). It is true that eqs. (15) and (16) follow from the form of $\dot{\bar{x}}$. We can apply Lemma 3.3 to obtain the desired result.

\section{APPLICATION}

We now apply the results of this work to study feedback control of protein production, where the feedback controller is implemented through sRNA-mRNA interference. This provides a scalable and experimentally easily tunable control system for several applications [5], [7], [13]. This controller is designed to make the concentration of a protein track a given reference signal while rejecting a perturbation on the translation rate [7]. The disturbance is due to the unavoidable time varying perturbation on ribosome concentration applied by by other processes in the cell. The sRNA controller is

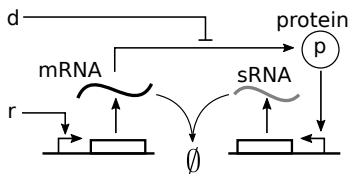

Fig. 1: A protein production process with a closed loop controller based on sRNA interference. Here $d$ represents that perturbation to ribosome concentration and $r$ represents a reference protein concentration.

based on the use of sRNA to degrade mRNA [13], [28], [29]. In order to regulate the expression of a protein, $p, p$ is used to induce the production of $s$, the sRNA molecule. $s$ and $m$ bind together and mutually degrade. By assuming that the molecular counts of all species are sufficiently large, we obtain the following nondimensionalized ODE model of the system [13]:

$$
\begin{aligned}
\dot{m} & =G r-G m s-\delta^{\prime} m, \\
\dot{s} & =G p-G m s-\delta^{\prime} s, \\
\dot{p} & =\gamma(m, d)-\delta p .
\end{aligned}
$$

where $\gamma(m, d)=\frac{m}{m+1+d}$. By tuning the biochemical parameters of the system $G$ and $\delta^{\prime}$ can be made very large. Specifically, $G$ can be made large by increasing the binding affinity of $m$ and $s$ by engineering the sRNA sequence [28]. $\delta^{\prime}$ can be made large by introducing auxiliary species, $m^{\prime}$ and $s^{\prime}$, which are engineered to bind to and mutually degrade $s$ and $m$ respectively. Here we assume that the concentrations of $m^{\prime}$ and $s^{\prime}$ are large. We assume that $\delta>0,0 \leq d \leq d_{\max }$, and $r_{\min } \leq r<1 / \delta$. To analyze (28) with the framework introduced in this paper, we let $\epsilon=1 / \delta^{\prime}$ and $\lambda=G / \delta^{\prime}$. System (28) then becomes

$$
\begin{aligned}
\epsilon \dot{m} & =\lambda r-\lambda m s-m, \\
\epsilon \dot{s} & =\lambda p-\lambda m s-s, \\
\dot{p} & =\gamma(m, d)-\delta p .
\end{aligned}
$$

(29) is of the form (25), allowing us to apply the theory of Section IV. In fact, system (29) has the asymptotic robust reference tracking property in the sense of Definition 2.1 with output $y=p$. To see this, observe that (29) is of the form (25) and satisfies Assumption 4.1. Hence, by Corollary 4.1 we have that (29) satisfies Definition 2.1 with output $y=p$.

To investigate how the results of this paper may inform design choices, we compute $\epsilon^{*}(\lambda)$ from Definition 2.1 for system (29). Figure 2 shows $\epsilon^{*}(\lambda)$. For three different points in the $(\lambda, \epsilon)$ parameter space we simulate tracking of a time varying signal $r(t)$, with $d(t)=0.5+0.1 \cos (0.5 t)$. By contrasting $\mathrm{A}$ and $\mathrm{B}$, we see that increasing $\lambda$ while keeping $\epsilon<\epsilon^{*}(\lambda)$ improves tracking performance, which is expected based on steady state analysis [13]. From $C$ we can see that for $\epsilon>\epsilon^{*}(\lambda)$ overshoot may emerge in $p(t)$, highlighting the practical importance of being able to pick $\epsilon$ sufficiently small when $\lambda$ is increased, as stated in Corollary 4.1. 

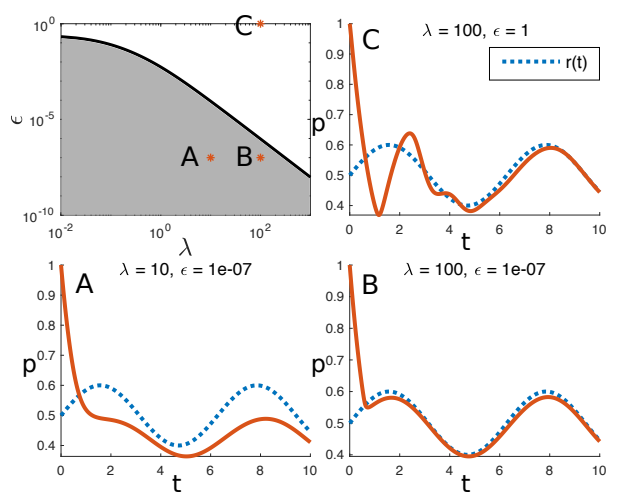

Fig. 2: Parameter space and simulations of the sRNA system. The gray region shows where we can certify tracking of slowly varying reference signals and rejection of slowly varying disturbances, i.e. the black line is $\epsilon^{*}(\lambda)$. To calculate $\epsilon^{*}$ we compute the following constants for system (29) with $\delta=1$ [30]: $c_{1}=1, c_{2}=2, c_{3}=2, b_{1}=1, b_{2}=1$, $b_{3}^{\prime}=2 \delta, v_{0}=1, L=\sqrt{2} \lambda, k_{1}=2, k_{2}=\lambda\left(1+d_{\max }\right)+\delta$, $k_{3}=1+d_{\max }$, and $k_{4}=4$. We use $d_{\max }=2, w_{0}=L(\lambda)^{2}$, and $r_{\text {min }}=0.25$. We then compute $\epsilon^{*}(\lambda)$ as defined in the proof of Lemma 3.1. A, B, and C show simulated trajectories of $p(t)$ for the system with the given parameters.

\section{CONCLUSIONS}

In this work we investigate principles for designing biomolecular feedback controllers that track slowly varying reference signals while rejecting slowly varying disturbance signals, with performance that becomes better as a "gain" parameter is made large. Due to linearized analysis being uninformative for the asymptotic problem, we resort to Lyapunov analysis and construct Lyapunov functions by exploiting timescale separation between the plant and controller. We provide explicit Lyapunov functions for a class of systems which are biologically relevant. We apply our theory to a controller based on mRNA-sRNA interference.

\section{APPENDIX}

\section{A. Proof of Lemma 3.1}

Our proof is inspired by those in [25], [31], but our assumptions yield conclusions that are uniform in $\lambda$.

Proof: Let $\nu\left(x, y_{b}\right)=(1-D) V(x)+D W\left(y_{b}\right)$ for $D \in(0,1)$. Observe that $\forall\left(x, y_{b}\right) \in S^{*}, \nu$ satisfies

$$
\begin{aligned}
& b_{1}(1-D)\|x\|_{2}^{2 a_{v}}+c_{1} D\left\|y_{b}\right\|_{2}^{2 a_{w}} \leq \nu\left(x, y_{b}\right) \\
& \leq b_{2}(1-D)\|x\|_{2}^{2 a_{v}}+c_{2} D\left\|y_{b}\right\|_{2}^{2 a_{w}},
\end{aligned}
$$

and so,

$$
\begin{array}{r}
\min \left\{b_{1}(1-D), c_{1} D\right\}\left(\|x\|_{2}^{2 a_{v}}+\left\|y_{b}\right\|_{2}^{2 a_{w}}\right) \leq \nu\left(x, y_{b}\right) \\
\leq \max \left\{b_{2}(1-D), c_{2} D\right\}\left(\|x\|_{2}^{2 a_{v}}+\left\|y_{b}\right\|_{2}^{2 a_{w}}\right) .
\end{array}
$$

Let us choose (see [25] for motivation) $D=\frac{v_{0}}{v_{0}+w_{0}}$. Observe that with this choice of $D S^{*}$ is the sublevel set $\nu\left(x, y_{b}\right) \leq$ $v_{0} w_{0} /\left(v_{0}+w_{0}\right)$. For each constant $u \in \mathcal{U}$, the dynamics of (6) in $\left(x, y_{b}\right)$ coordinates are

$$
\begin{aligned}
\dot{x} & =f\left(x, y_{b}+H(x), u\right), \\
\epsilon \dot{y_{b}} & =g\left(x, y_{b}+H(x), u, \lambda\right)-\epsilon \frac{\partial H}{\partial x} f\left(x, y_{b}+H(x), u\right) .
\end{aligned}
$$

Consider the derivative of $\nu$ on $S^{*} \backslash\left\{\left(x, y_{b}\right) \mid\|x\|_{2}^{a_{v}} \leq R(\lambda)\right\}$. Using (10) we have

$$
\begin{aligned}
& \dot{\nu}\left(x, y_{b}\right) \leq-(1-D) b_{3}^{\prime}\|x\|_{2}^{a_{v}} \\
&-\frac{D c_{3}}{\epsilon}\left\|y_{b}\right\|_{2}^{2 a_{w}}+(1-D) k_{1} k_{3}\|x\|_{2}^{a_{v}}\left\|y_{b}\right\|_{2}^{a_{w}} \\
& \quad+D k_{2} k_{4} L\left\|y_{b}\right\|_{2}^{a_{w}}\|x\|_{2}^{a_{v}}+D k_{3} k_{4} L\left\|y_{b}\right\|_{2}^{2 a_{w}}
\end{aligned}
$$

Since $S^{*}$ is compact we can write

$$
\begin{gathered}
\dot{\nu}\left(x, y_{b}\right) \leq-\frac{(1-D) b_{3}^{\prime}\|x\|_{2}^{a_{v}}}{2}-\frac{D c_{3}\left\|y_{b}\right\|_{2}^{2 a_{w}}}{2 \epsilon}-\frac{(1-D) b_{3}^{\prime}\|x\|_{2}^{2 a_{v}}}{2 \max _{x \in S^{*}}\|x\|^{a_{v}}} \\
-\frac{D c_{3}\left\|y_{b}\right\|_{2}^{2 a_{w}}}{2 \epsilon}+(1-D) k_{1} k_{3}\|x\|_{2}^{a_{v}}\left\|y_{b}\right\|_{2}^{a_{w}} \\
\quad+D k_{2} k_{4} L\left\|y_{b}\right\|_{2}^{a_{w}}\|x\|_{2}^{a_{v}}+D k_{3} k_{4} L\left\|y_{b}\right\|_{2}^{2 a_{w}} .
\end{gathered}
$$

Where we have used that $\forall x \in S^{*}, \frac{(1-D) b_{3}^{\prime}}{2 \max _{x \in S^{*}}\|x\|_{2}^{a_{v}}}\|x\|_{2}^{2 a_{v}} \leq$ $\frac{1}{2}(1-D) b_{3}^{\prime}\|x\|_{2}^{a_{v}}$. We therefore have that $\forall\left(x, y_{b}\right) \in S^{*} \backslash$ $\left\{\left(x, y_{b}\right) \mid\|x\|_{2}^{a_{v}} \leq R(\lambda)\right\}$

$$
\begin{aligned}
& \dot{\nu}\left(x, y_{b}\right) \leq-\frac{(1-D) b_{3}^{\prime}}{2}\|x\|_{2}^{a_{v}}-\frac{D c_{3}}{2 \epsilon}\left\|y_{b}\right\|_{2}^{2 a_{w}}-N^{T} M N \\
& \leq-\frac{(1-D) b_{3}^{\prime}\|x\|_{2}^{a_{v}}}{2}-\frac{D c_{3}\left\|y_{b}\right\|_{2}^{2 a_{w}}}{2 \epsilon}-\lambda_{\min }(M)\|N\|_{2}^{2},
\end{aligned}
$$

where

$$
M=\left[\begin{array}{cc}
\frac{(1-D) b_{3}^{\prime}}{2 \max _{x} \in S^{*}\|x\|^{a}} & -\frac{(1-D) k_{1} k_{3}+D k_{2} k_{4} L}{2} \\
-\frac{(1-D) k_{1} k_{3}+D k_{2} k_{4} L}{2} & \frac{c_{3} D}{2 \epsilon}-D k_{3} k_{4} L
\end{array}\right],
$$

and $N=\left[\|x\|_{2}^{a_{v}} \quad\left\|y_{b}\right\|_{2}^{a_{w}}\right]^{T}$. We need to find and $\epsilon^{* *}$ such that $\forall 0<\epsilon<\epsilon^{* *}, M \geq 0$. Noting that since $M_{11}>0$, by Sylvester's Criterion [26], $M>0$ if $\operatorname{det} M>0$, we find following condition

$$
\epsilon<\frac{\frac{D(1-D) c_{3} b_{3}^{\prime}}{4 \max _{x \in S^{*}}\|x\|^{a v}}}{\frac{(1-D) b_{3}^{\prime} D k_{3} k_{4} L}{2 \max _{x \in S^{*}}\|x\|^{a_{v}}}+\frac{\left[(1-D) k_{1} k_{3}+D k_{2} k_{4} L\right]^{2}}{4}}=: \epsilon^{*}
$$

which guarantees that $M>0$. We now consider the full case of $u(t)$ time varying. In $\left(x, y_{b}\right)$ coordinates, $(6)$ becomes

$$
\begin{aligned}
\dot{x} & =f\left(x, y_{b}+H(x), u\right)-\frac{\partial q_{1}}{\partial u} \dot{u}, \\
\epsilon \dot{y_{b}} & =g\left(x, y_{b}+H(x), u, \lambda\right)-\epsilon \frac{\partial q_{2}}{\partial u} \dot{u}-\epsilon \frac{\partial H}{\partial q} \frac{\partial q}{\partial u} \dot{u} \\
& -\epsilon \frac{\partial H}{\partial x}\left(f\left(x, y_{b}+H(x), u\right)-\frac{\partial q_{1}}{\partial u} \dot{u}\right) .
\end{aligned}
$$

From (32) and (33) and we have that

$$
\begin{array}{r}
\dot{\nu}\left(x, y_{b}\right) \leq-\frac{(1-D) b_{3}^{\prime}\|x\|_{2}^{a_{v}}}{2}-\frac{D c_{3}\left\|y_{b}\right\|_{2}^{2 a} w}{2 \epsilon}-\frac{\partial \nu}{\partial x} \frac{\partial q_{1}}{\partial u} \dot{u} \\
-\frac{\partial \nu}{\partial y_{b}} \frac{\partial q_{2}}{\partial u} \dot{u}+\frac{\partial \nu}{\partial y_{b}} \frac{\partial H}{\partial x} \frac{\partial q_{1}}{\partial u} \dot{u}-\frac{\partial \nu}{\partial y_{b}} \frac{\partial H}{\partial u} \dot{u}
\end{array}
$$

Therefore,

$$
\begin{gathered}
\dot{\nu}\left(x, y_{b}\right) \leq-\frac{(1-D) b_{3}^{\prime}\|x\|_{2}^{a_{v}}}{2}-\frac{D c_{3}}{2 \epsilon}\left\|y_{b}\right\|_{2}^{2 a_{w}}+\left\|\frac{\partial \nu}{\partial x}\right\|\left\|\frac{\partial q_{1}}{\partial u}\right\|\|\dot{u}\| \\
+\left\|\frac{\partial \nu}{\partial y_{b}}\right\|\left(\left\|\frac{\partial q_{2}}{\partial u}\right\|+\left\|\frac{\partial H}{\partial x}\right\|\left\|\frac{\partial q_{1}}{\partial u}\right\|+\left\|\frac{\partial H}{\partial u}\right\|\right)\|\dot{u}\| .
\end{gathered}
$$


By applying our bounds from (11), (14), as well as our bounds on $\left\|\frac{\partial q}{\partial u}\right\|$ and $\left\|\frac{\partial H}{\partial x}\right\|$, we obtain

$$
\begin{aligned}
\dot{\nu}\left(x, y_{b}\right) \leq(1-D) & \left(k_{1} B\|\dot{u}\|-\frac{b_{3}^{\prime}}{2}\right)\|x\|_{2}^{a_{v}} \\
+ & D\left(k_{4} \tilde{L}\|\dot{u}\|-\frac{c_{3}}{2 \epsilon}\left\|y_{b}\right\|_{2}^{a_{w}}\right)\left\|y_{b}\right\|_{2}^{a_{w}},
\end{aligned}
$$

where we have defined $\tilde{L}=B+L B+L^{\prime}$. Let $\bar{K}(\lambda)>$ 0 and define $\epsilon^{* *}:=\frac{k_{1} c_{3} B \bar{K}(\lambda)}{k_{4} b_{3}^{\prime} \tilde{L}}$ and $\epsilon^{* * *}=\min \left\{\epsilon^{*}, \epsilon^{* *}\right\}$. Let $S^{\prime}=\left\{\left(x, y_{b}\right) \mid\|x\|_{2}^{a_{v}} \leq R(\lambda),\left\|y_{b}\right\|_{2}^{a_{w}} \leq \bar{K}(\lambda)\right\}$. If $\epsilon<$ $\epsilon^{* * *}$ and $\forall t \geq 0,\|\dot{u}(t)\|<\frac{b_{3}^{\prime}}{2 k_{1} B}$, then $\forall\left(x, y_{b}\right) \in S^{*} \backslash$ $S^{\prime}, \dot{\nu}\left(x, y_{b}\right)<0$. Therefore, $\nu$ is decreasing on $S^{*} \backslash S^{\prime}$, and if $\left(x(0), y_{b}(0)\right) \in S^{*}$, eventually $\nu$ will be bounded by

$$
\nu^{\prime}:=\min _{a} a, \text { s.t. } S^{\prime} \subseteq\left\{\left(x, y_{b}\right) \mid \nu\left(x, y_{b}\right) \leq a\right\},
$$

i.e. $\forall\left(x, y_{b}\right) \in S^{*}, \quad \lim \sup _{t \rightarrow \infty} \nu(t) \leq \nu^{\prime}$. Using equation (30) We can find an upper bound on $\nu^{\prime}$ by solving $\nu^{\prime \prime}:=\min _{a} a$, s.t. $S^{\prime} \subseteq S^{\prime \prime}(a)$, where $S^{\prime \prime}=\left\{\left(x, y_{b}\right) \mid b_{1}(1-D)\|x\|_{2}^{2 a_{v}}+c_{1} D\left\|y_{b}\right\|_{2}^{2 a_{w}} \leq a\right\}$. By convexity of $S^{\prime \prime}$, the solution to this optimization problem is $\nu^{\prime \prime}=b_{1}(1-D) R^{2}(\lambda)+c_{1} D \bar{K}^{2}$. Hence, $\forall\left(x(0), y_{b}(0)\right) \in S^{*}$,

$$
\begin{aligned}
\limsup _{t \rightarrow \infty}\left(b_{1}(1-D)\|x\|_{2}^{2 a_{v}}\right. & \left.+c_{1} D\left\|y_{b}\right\|_{2}^{2 a_{w}}\right) \\
& \leq b_{1}(1-D) r^{2}+c_{1} D \bar{K}^{2} .
\end{aligned}
$$

Now consider the above result in $(x, z)$ coordinates. Let us denote the transformation from $(x, z)$ to $\left(x, y_{b}\right)$ by $\phi$, i.e. $\phi:(x, z) \rightarrow\left(x, y_{b}\right)$. Consider $\hat{\mathcal{P}}$ as defined in (17). It is true that $\hat{\mathcal{P}} \subseteq \phi^{-1}\left(S^{*}\right)$. Therefore we have the desired result.

\section{B. Proof of Lemma 3.3}

Proof: For all $\beta>\epsilon>0$ and sufficiently large $\lambda$, conditions 1 and 3 guarantee that $\exists R(\lambda)>0$ such that $\forall|x|>$ $R(\lambda),|\hat{f}(x, u, \lambda)|>\beta-\epsilon$. Let $R(\lambda)$ denote the set of such $r$ values for each $\lambda$. Now, let $r^{*}(\lambda)=\sup _{r \in R(\lambda)} r+1 / \lambda$. Observe that by condition $2 r^{*}(\lambda)$ is monotone decreasing. Since it is also bounded from below it has a limit. Suppose by way of contradiction that $\lim _{\lambda \rightarrow \infty} R(\lambda)>0$. Then, $\exists \bar{r}>0$ such that $\forall \lambda>\lambda^{*}, r^{*}(\lambda)>\bar{r}$. However, such an $\bar{r}$ cannot exist since that would mean that $\forall x>\bar{r}, \hat{f}(x, u, \lambda)<\beta-\epsilon$ which violates condition 3 . Hence, $\lim _{\lambda \rightarrow \infty} R(\lambda)=0$.

\section{ACKNOWLEDGMENT}

The authors thank Yili Qian for his valuable comments.

\section{REFERENCES}

[1] D. E. Cameron, C. J. Bashor, and J. J. Collins, "A brief history of synthetic biology," Nature Reviews Microbiology, vol. 12, pp. 381390, 042014.

[2] R. Kwok, "Five hard truths for synthetic biology," Nature News, vol. 463, no. 7279, pp. 288-290, 2010.

[3] P. E. M. Purnick and R. Weiss, "The second wave of synthetic biology: from modules to systems," Nature Reviews Molecular Cell Biology, vol. 10, pp. $410 \mathrm{EP}$-, 062009.

[4] D. Del Vecchio, "Modularity, context-dependence, and insulation in engineered biological circuits," Trends in Biotechnology, vol. 33, no. 2, pp. 111-119, 2015.

[5] Y. Qian, H.-H. Huang, J. Jiménez, and D. Del Vecchio, "Resource competition shapes the response of genetic circuits," ACS Synthetic Biology, vol. 6, no. 7, pp. 1263-1272, 072017.
[6] A. Gyorgy, J. Jiménez, J. Yazbek, H.-H. Huang, H. Chung, R. Weiss, and D. Del Vecchio, "Isocost lines describe the cellular economy of genetic circuits," Biophysical Journal, vol. 109, no. 3, pp. 639-646, 2015.

[7] H.-H. Huang, Y. Qian, and D. Del Vecchio, "A quasi-integral controller for adaptation of genetic modules to variable ribosome demand," Nature Communications, vol. 9, no. 1, p. 5415, 2018.

[8] A. Becskei and L. Serrano, "Engineering stability in gene networks by autoregulation," Nature, vol. 405, pp. 590 EP -, 062000.

[9] K. S. Nilgiriwala, J. Jiménez, P. M. Rivera, and D. Del Vecchio, "Synthetic tunable amplifying buffer circuit in e. coli," ACS Synthetic Biology, vol. 4, no. 5, pp. 577-584, 052015.

[10] C. Briat, A. Gupta, and M. Khammash, "Antithetic integral feedback ensures robust perfect adaptation in noisy biomolecular networks," Cell Systems, vol. 2, no. 1, pp. 15-26, 2016.

[11] V. Hsiao, E. L. C. de los Santos, W. R. Whitaker, J. E. Dueber, and R. M. Murray, "Design and implementation of a biomolecular concentration tracker," ACS Synthetic Biology, vol. 4, no. 2, pp. 150 161, 022015.

[12] G. Lillacci, S. K. Aoki, D. Schweingruber, and M. Khammash, "A synthetic integral feedback controller for robust tunable regulation in bacteria," 2017, bioRxiv:170951.

[13] Y. Qian and D. Del Vecchio, "Realizing 'integral control' in living cells: how to overcome leaky integration due to dilution?" Journal of The Royal Society Interface, vol. 15, no. 139, 2018.

[14] J. Ang and D. R. McMillen, "Physical constraints on biological integral control design for homeostasis and sensory adaptation," Biophysical Journal, vol. 104, no. 2, pp. 505-515, 2013.

[15] H. Steel and A. Papachristodoulou, "Design constraints for biological systems that achieve adaptation and disturbance rejection," IEEE Trans. Contr. Net. Syst., vol. 5, no. 2, pp. 807-817, 2018.

[16] E. D. Sontag, "Adaptation and regulation with signal detection implies internal model," Systems \& control letters, vol. 50, no. 2, pp. 119-126, 2003.

[17] F. Xiao and J. C. Doyle, "Robust perfect adaptation in biomolecular reaction networks," in IEEE Conf. Decision and Contr., 2018, pp. 4345-4352.

[18] K.-K. Young, P. Kokotovic, and V. Utkin, "A singular perturbation analysis of high-gain feedback systems," IEEE Trans. Automat. Contr. vol. 22, no. 6, pp. 931-938, 1977.

[19] P. Kokotović, H. Khalil, and J. O'Reilly, Singular Perturbation Methods in Control: Analysis and Design. Society for Industrial and Applied Mathematics, 2019/02/26 1999.

[20] R. Marino, "High-gain feedback in non-linear control systems," International Journal of Control, vol. 42, no. 6, pp. 1369-1385, 121985.

[21] Y. Qian and D. Del Vecchio, "A singular singular perturbation problem arising from a class of biomolecular feedback controllers," IEEE Contr. Syst. Letters, vol. 3, no. 2, pp. 236-241, April 2019.

[22] E. Bullinger and F. Allgöwer, "Adaptive -tracking for nonlinear higher relative degree systems," Automatica, vol. 41, no. 7, pp. 1191-1200, 2005.

[23] J. Huang and W. J. Rugh, "On a nonlinear multivariable servomechanism problem," Automatica, vol. 26, no. 6, pp. 963-972, 1990.

[24] _ "An approximation method for the nonlinear servomechanism problem," IEEE Trans. Automat. Contr., vol. 37, no. 9, pp. 1395-1398, 1992.

[25] A. Saberi and H. Khalil, "Quadratic-type lyapunov functions for singularly perturbed systems," IEEE Trans. Automat. Contr., vol. 29, no. 6 , pp. 542-550, 1984.

[26] R. A. Horn and C. R. Johnson, Matrix Analysis, 2nd ed. Cambridge University Press, 2012.

[27] F. Blanchini and E. Franco, "Structurally robust biological networks," BMC Systems Biology, vol. 5, no. 1, p. 74, 2011.

[28] S. M. Yoo, D. Na, and S. Y. Lee, "Design and use of synthetic regulatory small rnas to control gene expression in escherichia coli," Nature Protocols, vol. 8, pp. 1694 EP -, 082013.

[29] E. Levine, Z. Zhang, T. Kuhlman, and T. Hwa, "Quantitative characteristics of gene regulation by small rna," PLOS Biology, vol. 5, no. 9, pp. e229-, 082007.

[30] T. W. Grunberg and D. Del Vecchio, "Time-scale separation based design of biomolecular feedback controllers (extended version)," [online], 2019, available: http://hdl.handle.net/1721.1/120973.

[31] H. K. Khalil, Nonlinear Systems, 3rd ed. Upper Saddle River, NJ: Prentice Hall, 2002. 